Conclusion: Ocular toxicity remains as one of the most harmful and disabling adverse events in patients under $\mathrm{CQ} / \mathrm{HCQ}$ treatment. Higher age at diagnosis and higher age at beginning of treatment appear to be risk factors for maculopathy appearance. Screening protocols are well-assumed by patients and seemed to be helpful for preventing and early identifying events. CQ/HCQ usage in COVID-19 patients should be individualized, specially in older patients, and protocols involving ocular toxicity should be implemented in the follow-up of this population.

REFERENCES:

[1] T Dörner. Hydroxychloroquine in SLE: old drug, new perspectives. Nat Rev Rheumatol 6, 10-11 (2010)

[2] S Chowdhury, J Rathod, J Gernsheimer. A rapid systematic review of clinical trials utilizing chloroquine and hydroxychloroquine as a treatment for COVID19. Acad Emerg Med. 2020;27(6):493-504.

[3] J C S Yam, A K H Kwok. Ocular toxicity of hydroxychloroquine. Hong Kong Med J. 2006;12(4):294-304.

Disclosure of Interests: None declared

DOI: 10.1136/annrheumdis-2021-eular.3278

\section{AB0295 1 TREATMENT OF HIGH RISK/REFRACTORY OBSTETRIC ANTIPHOSPHOLIPID SYNDROME. A SINGLE CENTRE EXPERIENCE}

A. Hoxha ${ }^{1}$, P. Marson ${ }^{2}$, M. Favaro ${ }^{3}$, M. Tonello ${ }^{3}$, M. Zen ${ }^{3}$, T. Del Ross ${ }^{3}$, A. Calligaro ${ }^{3}$, A. Ruffatti ${ }^{4}{ }^{1}$ San Bortolo Hospital, Department of Medicine, Vicenza, Italy; ${ }^{2}$ University-Hospital of Padua, Padua, Italy, Apheresis Unit, Blood Transfusion Service, Padua, Italy; ${ }^{3}$ University Hospital of Padua, Padua, Italy, Department of Medicine, Rheumatology Unit, Padua, Italy; ${ }^{4}$ University Hospital of Padua, Padua, Italy, Department of Medicine, Padua, Italy

Background: The most efficacious strategy to manage pregnant patients with antiphospholipid syndrome (APS) who are at high risk of adverse pregnancy outcomes \pm refractory to conventional heparin/low-dose aspirin treatment is currently unknown $(1,2)$.

Objectives: The purposes of this study were to investigate the efficacy and safety of a second-line treatment protocol administered in addition to twice daily low molecular weight heparin and low-dose aspirin to pregnant patients affected with high-risk \pm refractory primary APS

Methods: Patients were included in the study if satisfying the following criteria were: 1) the presence of triple antiphospholipid antibody positivity (IgG/lgM anticardiolipin + IgG/IgM anti- $\beta 2$ Glycoprotein I antibodies + lupus anticoagulant), 2) previous thrombosis and/or a history of one or more early and severe pregnancy complications. The second-line treatment protocol included weekly plasmapheresis or immunoadsorption and fortnightly $1 \mathrm{~g} / \mathrm{kg}$ intravenous immunoglobulins. Results: Twenty-four pregnancies occurring between 2002 and 2019 in 19 primary APS patients, (mean age 35.1 $\pm 3.5 \mathrm{SD}$ ) were monitored. Triple antiphospholipid positivity was detected in all 19 cases $(100 \%)$. Seven of these women $(36.8 \%)$ had a history of thrombosis, five $(26.3 \%)$ one or more previous failed pregnancies associated to severe pregnancy complications and seven (36.8\%) both clinical criteria. Twentythree pregnancies $(95.8 \%$ ) produced live neonates (13 females and 10 males), all born between the $26^{\text {th }}$ and $38^{\text {th }}$ week of gestation (mean $33.6 \pm 3.5 \mathrm{SD}$ ); birth weight percentile was $35.8 \pm 24.1 \mathrm{SD}$ and mean Apgar score at $5 \mathrm{~min} 8.7 \pm 1.1 \mathrm{SD}$. Due to premature birth $\left(24^{\text {th }}\right.$ week) complicated by fetal sepsis, one pregnancy $(4.2 \%)$ had a negative outcome. During the treated pregnancy there were no episodes of thrombosis; there were five cases $(20.8 \%)$ of severe maternal complications during pregnancy or puerperium and four of fetal complications (16.6\%), all followed by complete recovery after delivery. No side-effects of the treatment were registered.

Conclusion: Given the high live birth rate and the safety associated to it, the second-line treatment protocol described here could be taken into consideration when the treatment of a high-risk APS pregnancy \pm refractory to conventional therapy is being evaluated.

REFERENCES:

[1] Tektonidou MG, et al. Ann Rheum Dis 2019;0:1-9. doi:10.1136/ annrheumdis-2019-215213

[2] Giacomelli et al. Autoimmun Rev. 2020;102738. doi.org/10.1016/j. autrev.2020.102738

Disclosure of Interests: None declared

DOI: 10.1136/annrheumdis-2021-eular.3449

\section{AB0296 \\ PASSIVE TRANSFER OF ANTI-SSA, ANTI-Ro52, AND ANTI-MITOCHONDRIAL M2 FROM INTRAVENOUS IMMUNOGLOBULIN PRODUCTS TO PATIENTS WITH RHEUMATIC DISEASES}

L. J. Yang ${ }^{1}$, H. G. Li ${ }^{1}$, A. Q. Zeng ${ }^{1}$, Z. M. Ouyang ${ }^{1}$, X. N. Wei ${ }^{1}$, Q. H. Li ${ }^{1}$, D.

H. Zheng ${ }^{1}$, L. Dai ${ }^{1}$, Y. Mo ${ }^{1} .{ }^{1}$ Sun Yat-Sen Memorial Hospital, Sun Yat-Sen

University, Department of Rheumatology, Guangzhou, China
Background: Passive transfer of ANA and anti-SSA has been reported in patients with common variable immunodeficiency disorder who received intravenous immunoglobulin (IVIG). IVIG is also recommended to treat some special or life-threatening rheumatic diseases.

Objectives: This study was aimed to explore whether any extractable nuclea antibodies (ENAs) were transferred to these rheumatic patients who received IVIG therapy.

Methods: IVIG products of three batches were tested for ANA by using indirect immunofluorescent assay, and for ENAs by using line immunoassay (LIA) and chemiluminescence immunoassay (CLIA). These IVIG products were administrated to rheumatic patients at a dose of $20 \mathrm{~g} / \mathrm{d} \times 3$ days (day1 to day3). Serum samples of these patients before IVIG (day0) and after IVIG (day4, day8, day10, day12, and more than one month) were tested by using LIA and CLIA. Anti-SSA was also detected using ELISA

Results: In these IVIG products, ANA was positive at a titer of 1:640 (cytoplasmic speckled) and 1:80 (speckled). Among 14 types of ENAs that could be tested using LIA, anti-SSA, anti-Ro52, anti-mitochondrial M2, and anti-centromere $B$ antibodies were clearly detectable in IVIG products (Table 1). Likewise, anothe assay CLIA also detected the same positive autoantibodies in these products. LIA showed the highest concentration in anti-mitochondrial M2, while CLIA showed the highest concentration in anti-mitochondrial M2 and anti-Ro52. One 31-yearold male patient who was diagnosed as SLE (Figure 1) and one 72-year-old male patients who was diagnosed as necrotizing myositis received these IVIG products. Anti-SSA, anti-Ro52, anti-mitochondrial M2, but not anti-centromere B, were positive in the day4 serum samples, although all of these antibodies were negative at baseline (day0). The concentration of these antibodies decreased gradually as days passed and became undetectable around one month after IVIG.

Table 1. The concentration of autoantibodies in intravenous immunoglobulin products

\begin{tabular}{lccccc}
\hline & anti-SSA & anti-Ro-52 & anti-mitochondrial M2 & anti-centromere B & Cut-off \\
\hline LIA(grey value) & $20 \pm 3$ & $28 \pm 3$ & $69 \pm 10$ & $19 \pm 4$ & $\geq 11$ \\
CLIA (U/ml) & $333 \pm 107$ & $444 \pm 86$ & $434 \pm 66$ & $390 \pm 89$ & $>20$ \\
ELISA (U/ml) & $90 \pm 13$ & NA & NA & NA & $>20$ \\
\hline
\end{tabular}

LIA, line immunoassay; CLIA, chemiluminescence immunoassay; ELISA, enzyme linked immunosorbent assay

Conclusion: This study preliminarily reported transient positivity of anti-SSA anti-Ro52, and anti-mitochondrial M2 in rheumatic patients maybe because the passive transfer of these antibodies from IVIG products to the patients, although the potential influence of this transfer on the rheumatic diseases remained unknown
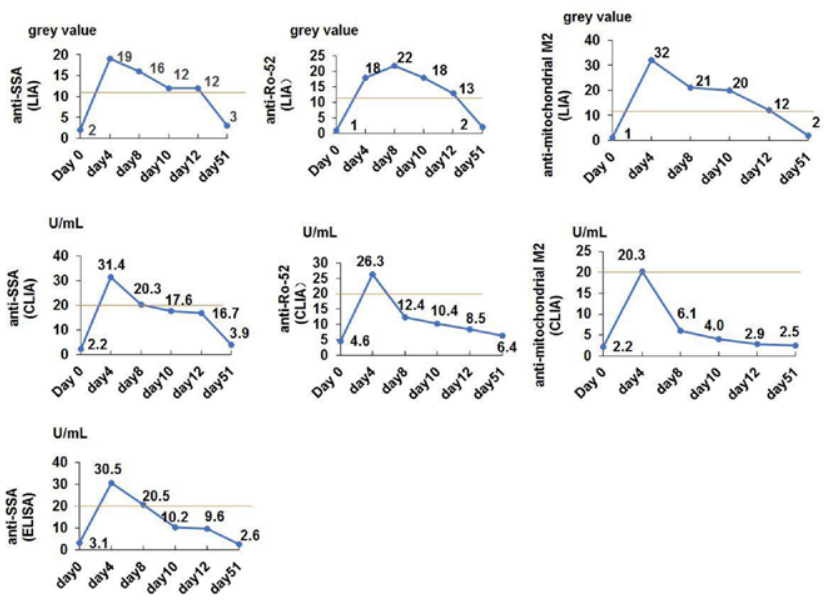

Figure 1. The concentration of autoantibodies in a 31-year-old male SLE patient receiving intravenous immunoglobulin at a dose of $20 \mathrm{~g} / \mathrm{d} \times 3$ days (day 1 to day3). Serum samples of these patients before IVIG (day0) and after IVIG (day4, day8, day10, day12, and day51) were tested by using line immunoassay (LIA) and chemiluminescence immunoassay (CLIA). AntiSSA was also detected using ELISA. The horizontal red lines were the corresponding cut-off values of each assay.

Disclosure of Interests: None declared DOI: 10.1136/annrheumdis-2021-eular.3544 